Cahiers d'études italiennes

\title{
In partibus infidelium
}

\section{Lise Bossi}

\section{OpenEdition \\ Journals}

Édition électronique

URL : http://journals.openedition.org/cei/208

DOI : $10.4000 /$ cei.208

ISSN : 2260-779X

\section{Éditeur}

UGA Éditions/Université Grenoble Alpes

Édition imprimée

Date de publication : 15 juillet 2009

Pagination : 197-209

ISBN : 978-2-84310-145-8

ISSN : $1770-9571$

Référence électronique

Lise Bossi, «In partibus infidelium », Cahiers d'études italiennes [En ligne], 9 | 2009, mis en ligne le 15 janvier 2011, consulté le 27 mars 2021. URL : http://journals.openedition.org/cei/208 ; DOI : https:// doi.org/10.4000/cei.208 


\title{
IN PARTIBUS INFIDELIUM
}

\author{
Lise Bossi \\ Université de la Sorbonne-Paris 4
}

Leonardo Sciascia a sans doute été l'un des premiers écrivains italiens d'après-guerre à dénoncer le rôle de l'Église, ou plutôt de l'institution ecclésiastique, dans la société et surtout dans la pratique politique de la nouvelle République italienne. Cela tient sans doute au fait qu'il est Sicilien et que la Sicile a souffert plus que toutes les autres régions de la Péninsule des excès d'une Inquisition toute-puissante sous la domination espagnole, des abus de pouvoir d'un clergé dévoyé, dénoncé, de Pirandello à Camilleri, à travers Le tonache di Montelusa , et surtout de la confusion des genres entre pouvoir politique et pouvoir religieux. Quoi qu'il en soit, nombreux sont les ouvrages où est stigmatisé le conflit d'intérêts, évident dans sa dénomination même, qui caractérise les objectifs du principal parti de gouvernement de la période qui nous intéresse : la Démocratie Chrétienne.

Cet aspect de l'œuvre de Sciascia lui a valu une réputation de mangiapreti et d'anti-clérical impénitent. Pourtant, son regard n'est pas que critique et son discours peut même se faire admiratif lorsqu'il lui est donné de rencontrer, rarement et le plus souvent par documents interposés, des hommes à la foi sincère dont la spiritualité profondément et évangéliquement vécue informe chacun des actes. C'est qu'il peut partager avec eux nombre de valeurs que les autres ont perdues. Cette communauté de valeurs a pu laisser croire à certains que Sciascia était passé de l'autre côté, du côté des fidèles. C'est l'avoir mal lu et avoir oublié que pour lui, les meilleurs chrétiens sont le plus souvent ceux que l'Église a relégués du côté des infidèles, in partibus infidelium.

I. Le tonache di Montelusa, sous-titre des nouvelles Difesa del Mèola, I fortunati et Visto che non piove... du recueil Scialle nero de Luigi Pirandello, où les ecclésiastiques sont dépeints sous un jour particulièrement sombre. Camilleri s'en souvient dans les portraits qu'il fait de ces mêmes hommes d'Église dans sa réinvention de Montelusa. 
Nous avons choisi de nous intéresser aux ouvrages de Sciascia dont les protagonistes sont des religieux et où le discours sur la religion prend le plus explicitement en compte tant les velléités temporelles de l'Église que les aspirations spirituelles de ceux qui, croyants ou non, au nom de la foi ou de la raison, luttent encore pour la vérité, la justice et la compassion. Nous examinerons donc, en tout premier lieu, Todo modo mais aussi Dalle parti degli infedeli et Morte dell'inquisitore et, pour finir, L'affaire Moro ${ }^{2}$ parce que si Aldo Moro n'était pas un homme d'Église Sciascia a écrit sur lui et pour lui un livre religieux.

Même si Sciascia a dit que Todo modo est la préfiguration littéraire sinon le facteur déclenchant de l'affaire Moro, ce roman est une fiction. L'écrivain y met en évidence les troubles liens que l'Église entretient avec le pouvoir et les hommes politiques. Ces derniers, au prétexte de participer à des exercices spirituels, se réunissent chaque année dans un lieu où tout se décline sous le signe de l'ambiguïté et du dédoublement, depuis le bâtiment lui-même, à la fois ermitage et hôtel, en passant par Don Gaetano, le maître des lieux, un prêtre qui a lu tous les livres et porte les lunettes du Diable, jusqu'aux retraitants que le narrateur de Sciascia compare tantôt à des animaux voraces solidement installés sur " une avalanche de nourriture en décomposition" ( $T M$, p. 44) dans laquelle ils s'agitent pour manger plus, plus vite et mieux que les autres, tantôt au radeau de la Méduse (ibidem, p. 48-49) : la "grande bouffe " des suppôts du pouvoir devenant une sorte de festin des Atrides où les hommes dévorent ou font dévorer leurs semblables pour assurer leur propre ignominieuse survie. C'est d'ailleurs Don Gaetano qui lui dévoile la vraie nature des présents et de leurs " activités":

- Vous vous trompez grandement en croyant que ceci est une espèce de congrégation : c'est un nœud de vipères. - Elles sont en train de se mordre entre elles ? - Vous ne vous en rendez donc pas compte? (Ibidem, p. 76)

Le problème est de savoir pourquoi Don Gaetano, c'est-à-dire pourquoi l'Église, cautionne et favorise les agissements de cette classe politique qui a fait de l'abus de biens sociaux, de l'hypocrisie et du mensonge un signe de reconnaissance sinon une règle de vie. L'explication la plus simple, la première qui vient à l'esprit lorsque l'on examine l'histoire conjointe de l'Église et de l'État italien, c'est que l'Église à travers les hommes du

2. Respectivement, L. Sciascia, Todo modo, Torino, Einaudi, I974; id., Dalle parti degli infedeli, Palermo, Sellerio, 1979 ; id., Morte dell'inquisitore, Bari, Laterza, 1964 ; id., L'affaire Moro, Palermo, Sellerio, 1978. Ci-dessous TM, DPDI, $M D I$ et $A M$. 
parti qui se réclame d'elle et dont elle a voulu qu'il soit le parti de tous les catholiques, entend ainsi reprendre à l'État spoliateur du Risorgimento ce pouvoir temporel et cette influence sur la société italienne qu'elle a perdus après I870. Et comme elle n'a pas réussi à détruire l'État, en bloquant ses institutions parlementaires grâce au Non expedit ${ }^{3}$ ou en attirant sur lui les foudres des grandes puissances catholiques - ce qui a eu pour seul effet de pousser l'Italie dans les bras des puissances protestantes en plein Kulturkampf-elle a choisi de se réconcilier avec lui, Patto Gentiloni ${ }^{4}$ à l'appui, pour préserver ses intérêts et ceux de ses fidèles face à la montée savamment orchestrée par Giolitti des idées marxistes et des protestations sociales.

La stratégie ayant fait ses preuves, l'Église est passée de la réconciliation à la conciliation, du Patto Gentiloni aux Patti Lateranensi et, par la même occasion, du parlementarisme au fascismes.

Cette belle indifférence à la nature des régimes dont elle reçoit garanties et pouvoir lui a permis de bénéficier de la transposition pure et simple des Accords de Latran dans l'article 7 de la Constitution de la toute nouvelle République italienne opérée par un groupe de travail où siégeaient des communistes avant d'excommunier ces derniers et de faire siens les idéaux de la guerre froide au nom du combat contre l'Antéchrist. C'est d'ailleurs au nom de ce même combat qu'elle a vivement encouragé son clergé à voter et surtout à faire voter pour les représentants de ce qui est désormais son parti, comme Sciascia le montre et le dénonce non seulement dans Dalle parti degli infedeli, où Monseigneur Ficcara qui entend s'occuper de ses ouailles du seul point de vue de sa mission pastorale se voit réprimandé puis écarté, mais aussi dans de nombreux autres textes, comme par exemple dans le récit La zia d'America', où même la communauté italo-américaine est mise à contribution pour prêcher la bonne parole en soutenant De Gasperi et les siens.

Ce n'est cependant pas ce retour en force de l'Église dans les rouages du pouvoir temporel en tant que tel qui inquiète Sciascia, c'est plutôt ce qu'il croit alors être, et qui est peut-être effectivement pour certains groupes

3. Né eletti, né elettori, pena la scomunica, document publié le I3 octobre I874 par lequel le pape Pie IX interdit aux catholiques toute participation à la vie politique du Royaume d'Italie.

4. Signé en 1913, il peut être considéré comme une préfiguration des accords de Latran, en particulier dans son heptalogue où le Royaume d'Italie s'engage à préserver les intérêts et les valeurs de l'Église et des catholiques.

5. Très représentatives de ce goût pour le consensus, les pages où Sciascia évoque ces divers accords " contre nature ", in $1912+I$, Milano, Adelphi, I986, p. 2I-22.

6. La zia d'America in Gli zii di Sicilia, Torino, Einaudi, 1958. 
politico-religieux tels Comunione e liberazione ou l'Opus dei, le but ultime de cette mainmise sur la chose publique.

Dans la fiction littéraire, c'est bien sûr Don Gaetano qui est chargé d'exposer, sous forme de boutade d'abord, ce que serait la fonction première des hommes de la Démocratie chrétienne dans une telle perspective :

Messieurs - dit Don Gaetano au ministre et au président - j'espère que vous ne me m’infligerez pas la douleur de me dire que l'État existe encore... À mon âge et avec toute la confiance que j'ai eue en vous, ce serait une révélation insupportable. J'étais tellement sûr qu'il n'existait plus. (TM, p. IIs)

Car Don Gaetano se réclame de l'Évangile de Luc : " Je suis venu apporter le feu sur la terre et que puis-je faire s'il brûle déjà »( $T M$, p. IO3). Ce feu, la classe dirigeante qui gouverne et commande " avec [sa] bénédiction sinon avec [son] mandat " (TM, p. IO4) l'a allumé et l'entretient et c'est pourquoi il la soutient, car elle contribue à détruire ce monde encore accroché à la raison et aux principes des Lumières auxquels certains de ceux qui ont gouverné l'Église du Christ ont commis l'erreur de s'identifier. Alors que, selon Don Gaetano, le triomphe de l'Église sera une épiphanie en forme d'apocalypse à laquelle vont contribuer les découvertes de la science, la satiété, la faim et l'ignorance. En effet, elles vont à tel point faire croître l'angoisse, celle que Pascal déjà éprouvait face à l'univers, qu'elles vont nous conduire à l'aube de l'époque la plus chrétienne que le monde puisse connaître. "Et l'angoisse cosmique, conclut-il, ne sera rien comparée à la peur que l'homme aura de lui-même et de ses semblables " (TM, p. I05). Ainsi, peu importent les moyens, les hommes, les partis, qui ne sont que des rouages interchangeables ${ }^{7}$, pourvu que ce triomphe puisse avoir lieu. Car comme le dit Don Gaetano « Dieu existe donc tout est permis " $(T M$, p. 76$)$ dans la mesure où Dieu est la cause première de toute chose et où ce qui découle de lui, l'univers tout entier donc, est justifié par son existence y compris le délit, la douleur et la mort. Y compris l'injustice et l'iniquité. C'est que, pour citer encore Don Gaetano :

La grandeur de l'Église, sa transhumanité, réside dans le fait qu'elle réalise la consubstantiation d'une espèce d'historicisme absolu : l'inévitable et précise nécessité, l'utilité certaine, de tout événement intérieur en rapport avec le monde, de chaque individu qui la sert et en porte témoignage, de chaque élément de sa hiérarchie, de tout changement ou succession... (TM, p. 48)

7. On retrouve cette idée en particulier in $T M$, p. 4I et IO2.

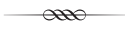


Pour les lecteurs de Sciascia, ces définitions de l'Église et de son rôle dans Todo modo font irrésistiblement penser au passage de $I l$ contesto ${ }^{8}$, que nous allons tenter de résumer ici, où le président Riches parle de la justice par décimation ; celle où l'individu répond de l'humanité et l'humanité de l'individu parce que poursuivre le coupable, les coupables est devenu impossible car il ne s'agit plus de chercher une aiguille mais bien un brin de foin dans la botte de foin. De ce fait il n'y a plus d'individu ou de responsabilité individuelle et le déshonneur et le délit doivent être rendus à la multitude. C'est là le seul moyen de combattre

ces délits contre la légitimité de la force que seule la force, en passant du côté du délit, peut effacer comme délits et s'incorporer sous la forme, inaltérablement prête à la recevoir, d'une entrée de Dieu dans le monde. La seule entrée que le monde permette à Dieu... (ibidem, p. 92).

Si nous rapprochons cette image de l'administration de la justice, c'est-àdire, pour Sciascia, de l'injustice, telle qu'elle est pratiquée par les représentants d'un État que Don Gaetano méprise tout en le cautionnant, si nous la rapprochons de la définition de l'Église comme consubstantiation d'un historicisme absolu où l'antique prédestination et le moderne déterminisme s'associent pour aboutir à une espèce d'auto-référentialité, nous sommes amenés à penser que l'Église - comme institution au service du dieu de la force - est parvenue à intégrer au sein d'un ordre supérieur la justification des délits sur lesquels l'exercice de la force est fondé. En d'autres termes, le rapport consubstantiel entre l'Église et l'Histoire tel qu'il est défini par Don Gaetano, c'est que l'Histoire est devenue l'histoire d'un pouvoir qui se prétend légitimé par une volonté supérieure dans une chaîne de causalité prédéterminée. Et c'est pour briser cette chaîne de causalité que le narrateur de Todo modo décide d'accomplir un acte de liberté et de tuer Don Gaetano, avec la bénédiction de Kant.

Pour comprendre l'acte du narrateur, il faut prendre conscience qu'avec Todo modo, Sciascia a travaillé sur deux plans qui comme toujours chez lui sont totalement imbriqués, le plan narratif et le plan philosophique, l'un reflétant métaphoriquement l'autre. Sur le plan narratif, ce récit qui apparaît comme la dernière des fictions policières de Sciascia après Il giorno della civetta, A ciascuno il suo ${ }^{9}$ et $\mathrm{Il}$ contesto, se présente en fait comme un anti-roman policier où l'enquêteur n'est plus un représentant de la loi et où il est même devenu un assassin. L'explication de ce renversement est à

8. Torino, Einaudi, I97I.

9. Respectivement, Il giorno della civetta, Torino, Einaudi, I961 et A ciascuno il suo, Torino, Einaudi, 1966. 
rechercher dans le passage de $I l$ contesto que nous citions précédemment. Au cours de sa diatribe en faveur de la justice par décimation Riches y dit à Rogas :

Votre métier mon cher est devenu ridicule. Il présuppose l'existence de l'individu et il n'y a pas d'individu, il présuppose l'existence de Dieu, le dieu qui aveugle les uns et illumine les autres, le dieu qui se cache, et il est resté caché si longtemps que nous pouvons présumer qu'il est mort ${ }^{\mathrm{t}}$.

Pour mieux comprendre le sens et l'importance de l'articulation entre l'existence de Dieu et le métier d'enquêteur, il faut revenir à ce que Sciascia a écrit à propos du roman policier :

Dans sa forme la plus originale et la plus autonome, le roman policier présuppose une métaphysique : l'existence d'un monde " au-delà du physique ", de Dieu, de la Grâce - et de cette Grâce que les théologiens appellent illuminante. De la Grâce illuminante l'enquêteur peut même se considérer comme le porteur, à l'instar de sainte Lucie dans la Divina Commedia ("Lucia, nimica di ciascun crudele »). L'incorruptibilité et l'infaillibilité de l'enquêteur, sa vie presque ascétique [...], le fait qu'il ne représente pas la loi officielle mais la loi dans l'absolu, sa capacité de lire le délit dans le coeur humain outre que dans les choses, c'est-à-dire dans les indices, et de le pressentir, l'investissent d'une lumière métaphysique, en font un élu. Et ce n'est pas un hasard si l'histoire du roman policier, la naissance de l'enquêteur, a dans la Bible ses premières origines" ${ }^{\text {. }}$

C'est à la fin de $19 I 2+I$, addition sous laquelle se cache superstitieusement l'année où le pacte Gentiloni a été signé, que se précise définitivement le système d'équivalence de Sciascia. Il y est fait allusion à un récit de Huxley « que l'on pourrait qualifier de policier : à ceci près que n'y est pas prononcé le nom de Dieu, que tout récit policier prononce d'ordinaire en l'englobant dans le mot justice ${ }^{12}$ ».

Ce qui signifie que Dieu n'est pas nommé voire qu'il est nié dès lors que justice et vérité sont absentes. Il n'est pas étonnant, du coup, que Todo modo soit la dernière des enquêtes proprement policières de Sciascia ${ }^{13}$, dès lors que la conscience du bien et du mal et, donc, les sentiments de culpabilité et de responsabilité qui en découlent, en ont complètement disparu. Sans doute parce que l'Église y prend clairement le parti du dieu de la force contre celui du dieu qui se cache ; le parti du dieu borgésien,

Io. Il contesto, p. 90.

II. Cruciverba, Torino, Einaudi, I983, p. 218. Il est bien sûr question ici de l'épisode où Daniel confond les vieillards qui accusaient mensongèrement Suzanne.

I2. $I 9 I 2+I$, p. 86 .

13. En fait Sciascia écrira une toute dernière enquête policière, en forme de testament désespéré, Una storia semplice, Milano, Adelphi, 1989, dont l'exergue est une citation du Justice de Friedrich Dürrenmatt : "Ancora una volta voglio scandagliare scrupolosamente le possibilità che forse ancora restano alla giustizia. " 
indifférent à la destinée des hommes, contre celui de ceux qui voient « le siècle et le monde régis par la loi et la loi par Dieu même [s'ils lui donnent] d'autres noms " (TM, p. 77) : comme justice, ou vérité, justement.

Autrement dit, si Sciascia abandonne le roman policier après cet antiroman policier qu'est Todo modo c'est qu'il a voulu marquer, sur le plan philosophique cette fois, que la justice et la vérité n'existent plus dans le monde de Don Gaetano et de ses protégés et qu'un genre littéraire qui a pour vocation de les restaurer est désormais tout aussi inutile et inopérant que le métier d'enquêteur.

C'est pourquoi il s'attache à les chercher dans une autre dimension qui est celle de la littérature et plus généralement de la chose écrite où elles sont contenues et préservées. La dimension où l'écrivain catholique Manzoni est allé avant lui retrouver, dans sa Storia della colonna infame, la trace d'une injustice qui pouvait être vue par ceux-là même qui la commettaient. Manzoni qui a "inventé » avec cet ouvrage méconnu le genre de l'enquête philologique comme réparation littéraire, fondée sur des présupposés éthiques, des injustices de l'histoire. Et c'est à ce nouveau genre que Sciascia se consacre désormais.

Il semble donc que l'on puisse en déduire que, comme dans les romans policiers où Son existence est postulée à travers la possible distinction entre le bien et le mal et la possible restauration du bien et donc de la justice, l'idée de Dieu est présente dans le principe des enquêtes philologiques que Sciascia conduit selon des présupposés voisins de ceux de Manzoni. Mais que l'on ne s'y trompe pas, si elle est présente c'est - la différence est de taille - dans les mots " vérité » et " justice » sur lesquels ces enquêtes se fondent et cela ne veut pas dire qu'à l'instar de son modèle littéraire Sciascia a l'intention ou la tentation de se convertir. En fait, plus que d'une idée de Dieu, il s'agit, pour Sciascia, de l'idée que la raison peut se faire de la vérité et de la justice à travers la connaissance du bien et du mal dont elle trouve en elle-même une définition. La volonté de faire le bien ne dépend plus, dans cette perspective, d'une référence à une entité transcendante révélée mais d'un devoir que la raison s'impose à elle-même et impose au sujet sous la forme d'un impératif catégorique. La conclusion kantienne de Todo modo, qui clôt la série des romans policiers et ouvre celle des enquêtes philologiques, laisse peu de doutes à ce sujet.

C'est dans cette perspective, plus rationnelle que métaphysique, au sens commun du terme, qu'il faut lire les deux ouvrages, que l'on peut aussi considérer comme des hommages, consacrés à des hommes d'Église. Le premier, Dalle parti degli infedeli, est une réflexion-commentaire sur les lettres échangées entre Angelo Ficcara, évêque de Patti, et la Sacra 
congregazione concistoriale. Ces lettres, insiste Sciascia, ont l'intérêt d'appartenir à une époque proche de la nôtre et elles montrent un juste, dont il entend prendre la défense, victime d'une persécution entièrement fondée sur le mensonge (DPDI, p. 79). Le point de départ est une circulaire de la Sacra congregazione où il est dit que "l'attention des évêques doit se porter sur le devoir pastoral d'instruire les fidèles à l'occasion et à proximité d'événements particuliers dont dépend le destin du Pays, particulièrement dans l'ordre moral et social ». Et l'on peut jusqu'ici admettre que l'Église se préoccupe de l'ordre moral même si elle empiète un peu trop sur l'ordre social. Mais, après tout, l'un découle souvent de l'autre. La suite en revanche est à la fois beaucoup plus claire et beaucoup moins défendable :

Ce ministère veut encore répéter qu'il est nécessaire de faire part aux fidèles de façon claire et répétée $[s i c]$, à l'occasion des élections législatives ou administratives, des élections nationales ou régionales des règles suivantes :

I) Considérant les périls auxquels sont exposés la religion et le bien public dont la gravité exige la collaboration unanime des honnêtes gens, tous ceux qui ont le droit de vote $[\ldots]$ sont en conscience strictement obligés d'en faire usage ;

2) Les catholiques ne peuvent donner leur voix qu'à ces candidats ou à ces listes de candidats dont on a la certitude qu'ils respecteront et défendront l'observance de la loi divine et les droits de la religion et de l'Église dans leur vie privée et dans la vie publique $[\ldots]$ (DPDI p. I4);

et ainsi de suite, comme pour mieux prouver que la réalité dépasse décidément toujours la fiction. Or, qu'a osé faire l'évêque de Patti : il a séjourné dans sa ville natale de Canicattì pendant la préparation des élections au lieu d'apporter sa contribution à la Démocratie Chrétienne et il a renvoyé à son vicaire une commission de femmes venues se plaindre de la conduite indigne du clergé. C’est pourquoi le représentant de la Démocratie Chrétienne, qui dénonce ces si graves manquements aux autorités politiques et religieuses dont il estime dépendre, abandonne son poste de combat non sans blâmer grandement " tous ceux qui ont trahi la Démocratie Chrétienne et donc l'Église " (DPDI, p. I6). Pour Monseigneur Ficcara c'est le commencement des ennuis et c'est désormais, selon Sciascia, à un véritable procès inquisitorial qu'il va être soumis ( $D P D I, \mathrm{p}$. 29). On va lui demander des comptes sur la conduite de ses prêtres, sur la diffusion du communisme, du protestantisme et de la franc-maçonnerie dans son diocèse, ainsi que sur le comportement de ses ouailles, sous les espèces de ce que Sciascia appelle ironiquement les âmes votantes. Puis on va lui apprendre qu'il souffre d'une baisse de ses facultés physiques et même mentales au point qu'on va lui adjoindre un auxiliaire car « l'époque exige 
une action pastorale prompte énergique et efficace " (DPDI, p. 37) et pour finir on décide de le promouvoir archevêque de Leontopoli de Augustamnica, in partibus infidelium, dans les contrées des infidèles ou, plus littéralement, du côté des infidèles. Et l'on goûtera le double sens que revêt cette expression dans un tel contexte. Car Monseigneur Ficcara est bien un infidèle, à l'Église du dieu de la force et à la Démocratie Chrétienne, et il dérange en conservant aux yeux de ses ouailles :

le prestige de l'évêque qui se contente d'être évêque, l'influence de celui qui ne fait pas de politique, qui voit la politique comme autre et comme autre le parti démocrate chrétien et qui, sans préjugés religieux et en toute bonne foi, laisse que chacun soit libre d'être avec la D.C., contre elle ou en dehors d'elle. (p. 5I)

Mais pour cette même raison l'ampleur et la teneur des réseaux d'intérêts, la profondeur des racines et la force débordante du phénomène démocrate-chrétien lui échappent, comme lui échappe la solidarité qui conduit l'Église à s'identifier, dans ces années de guerre froide, à un parti politique. (p. 42)

C'est que Monseigneur Ficcara croit en Dieu et pas dans le dieu qui décide que les personnes en bonne santé sont malades et que les justes doivent démissionner; dans le Dieu de la vérité et dans le Dieu de la justice (p. 38). Et Sciascia imagine sa colère, sa révolte, et comprend qu'il ne puisse se résigner à ce que de l'Église, de son Église, lui viennent le mensonge et l'iniquité. (p. 39)

Que la compréhension et, disons-le, le respect de Sciascia pour cet homme d'Église, ou plutôt pour cet homme de foi, repose non pas sur une quelconque tentation religieuse ou sur une aspiration à la transcendance mais sur ces valeurs de justice, de vérité, de tolérance et de charité que, n'en déplaise à Don Gaetano, le christianisme et les Lumières ont en commun, cela est confirmé par Morte dell'inquisitore, l'autre enquête philologique dont le protagoniste est un religieux. Une enquête qui est en fait la première dans le temps, dont Sciascia nous dit :

C'est, parmi les ouvrages que j'ai écrits, celui qui m'est le plus cher, le seul que je relise et à propos duquel je me tourmente encore. La raison en est que c'est effectivement un livre inachevé, que je ne finirai jamais, que je suis toujours tenté de réécrire et que je ne réécris pas dans l'attente de découvrir encore quelque chose : un nouveau document, une nouvelle révélation qui se dégagerait des documents que je connais déjà, quelque indice qu'il m’arriverait de découvrir entre veille et sommeil comme cela advient au Maigret de Simenon lorsqu'il est pris par une enquête. (p. 38)

Il convient déjà de noter que, avec cette déclaration, nous sommes sans conteste dans la double dimension de l'enquête et de la réparation littéraire manzonienne, c'est-à-dire dans la sphère où l'on présuppose l'existence de Dieu qui est lui-même englobé dans le mot justice. Pour le reste, l'histoire de fra Diego aurait pu être celle de Robin des bois s'il 
n'avait vécu en Sicile au milieu du XVII ${ }^{\mathrm{e}}$ siècle, au moment où les nobles espagnols et la Sainte Inquisition étaient tout-puissants. Pour avoir sans doute professé une hérésie qui, sans participer d'un mouvement religieux déjà défini, faisait appel à certains principes sociaux de l'Évangile ${ }^{\mathrm{I} 4}$ et pour en être arrivé de ce fait à la conviction non pas, explicitement, que Dieu était injuste mais qu' "il ne pouvait pas, sans être injuste, accepter l'injustice du monde » (p. 227), ce moine a été confié au Grand Inquisiteur en personne. Celui-ci s'est occupé de lui si paternellement et avec tant d'insistante bonté que fra Diego l'a tué en l'assommant avec ses propres chaînes. Pour le double crime d'hérésie et de meurtre sur la personne d'un représentant de l'Inquisition, il est condamné au bûcher, non sans avoir préalablement refusé de se rendre aux raisons de ses saints contradicteurs. De ces derniers Sciascia dit qu'ils resteront dans l'histoire du déshonneur humain pour leur intolérance et de fra Diego que c'est un homme qui affirme la dignité et l'honneur de l'homme, la force de la pensée, la ténacité de la volonté et la victoire de la liberté (p. 2II). Il ne fait donc aucun doute que, ici comme ailleurs, les sentiments de Sciascia vis-à-vis de l'Église ou plus exactement des institutions ecclésiastiques sont on ne peut plus tranchés et négatifs. Mais son intérêt et son attachement jamais démentis pour fra Diego et son histoire sont-ils seulement pour Sciascia l'occasion d'une charge contre les excès de l'Inquisition et l'intolérance de l'Église ? L'occasion d'un hommage à ceux pour qui la justice et la vérité ont été un souci constant, religieux ou pas, même si les religieux ont plus de mérite à avoir conservé ces valeurs dans une Église qui ne les a pas ou qui ne les a plus ? Ou bien y a-t-il pour finir une part de l'écrivain qui s'identifie à fra Diego lorsqu'il formule

l'hypothèse que, de la révolte contre l'injustice sociale, contre l'iniquité, contre l'usurpation des biens et des droits, [fra Diego] soit arrivé, au moment où il entrevoyait comme irrémédiable et sans espoir sa propre défaite et en identifiant son destin au destin de l'humanité, sa tragédie à la tragédie de l'existence, à accuser Dieu. Non pas à le nier mais à l'accuser? (p. 226)

Manzoni lui-même n'en est-il pas venu à dire, à propos du comportement des juges qui ont condamné des innocents pour sorcellerie dans les procès contre les untori, qu' « en cherchant un coupable à qui s'en prendre

I4. Ibidem, p. 22I. Sciascia suppose que c'est justement cette référence à l'Évangile qui est à l'origine de la relative indulgence de l'Inquisition envers fra Diego lors de sa première arrestation (ibidem, p. 199-200). 
à raison, l'esprit se trouve conduit avec effroi à hésiter entre deux blasphèmes, qui sont deux délires : nier la providence ou l'accuser ${ }^{15}$ » ?

D'ailleurs, toute enquête tendant à rendre justice à la victime contre le bourreau, dès lors qu' elle implique la contemplation de l'injustice en train de se perpétrer n'amène-t-elle pas l'enquêteur à se tourner vers Dieu et à lui demander raison ? C'est bien là le nœud du rapport que le laïc et le rationaliste Sciascia peut avoir non pas avec le dieu de la force mais avec le dieu caché, celui qui est englobé dans le mot justice. Parce que, comme il le fait dire à Don Gaetano :

Le laïcisme n'est que le revers d'un excès de respect pour l'Église et pour nous autres prêtres. Vous nous appliquez et vous appliquez à l'Église une sorte d'aspiration à la perfection : mais en restant confortablement en-dehors d'elle. Pour vous répondre, nous ne pouvons que vous inviter à venir à l'intérieur et à essayer, avec nous, d'être imparfaits. (TM, p. 39)

Et c'est pourquoi, selon la vision de l'Église que défend Don Gaetano :

Les bons prêtres sont les mauvais. La survivance et, plus que la survivance, le triomphe de l'Église à travers les siècles, sont dus plus aux mauvais prêtres qu'aux bons. C'est derrière l'image de l'imperfection que vit l'idée de la perfection : le prêtre qui contrevient à la sainteté ou qui, dans sa façon de vivre, va jusqu’à la dévaster ne fait que la confirmer, l'élever et la servir. (TM, p. 47-48)

C'est, à l'inverse, pourquoi Sciascia s'intéresse à ceux qui, dans la classification de Don Gaetano, sont les mauvais prêtres, ceux que l'Église a rejetés, exclus et tenté de faire disparaître, physiquement, par la torture et le bûcher, comme le fra Diego de Morte dell'inquisitore ou moralement, par la persécution mentale et la relégation In partibus infidelium, comme Monseigneur Ficcara. Et il ne le fait pas, comme le prétendrait Don Gaetano, parce qu'il voudrait réprimer en lui tout ce qui le porterait vers l'Église (TM, p. I06), même s'il avoue ressentir, de façon très contrariée et avec une grande méfiance, une nostalgie pour une certaine Église et pour son mystère : «Cette religion m'est toujours apparue comme un port sûr, un lieu et un refuge, une plage tranquille sur laquelle m'endormir, moi qui ne suis qu'un mécréant ${ }^{16}$. "Un mécréant, le mot est clair ; et qui a choisi de ne pas dormir, de ne pas se reposer, car il pense comme

15. Ibidem. On notera que les termes de la phrase, tirée de l'introduction à la Storia della colonna infame, sont repris mot à mot par Sciascia, qui établit ainsi une continuité dans l'horreur entre le supplice de fra Diego et celui des supposés untori milanais mais aussi, dans la dénonciation de cette horreur, une continuité entre Manzoni et lui.

I6. L. Sciascia, La Sicile comme métaphore, Paris, Stock, I979, p. III. Phrase curieusement absente de l'édition italienne (La Sicilia come metafora, Milano, Mondadori, 1979). 
le peintre de Todo modo que "beaucoup de choses en nous, que nous croyons mortes, sont comme dans une vallée du sommeil : une vallée sans aménité, une vallée qui n'a rien d'ariostesque. Et [que] sur leur sommeil la raison doit toujours veiller" $(T M$, p. 26). En fait, la confusion quant à une éventuelle tentation catholique chez Sciascia vient de ce qu'il a, outre la préoccupation d'une claire définition du bien et du mal - qui est la condition nécessaire au triomphe de la justice -, au moins deux sentiments en commun avec les croyants sincères : la pensée de la mort et la pitié. S'agissant de la pensée de la mort, elle est, selon l'écrivain, l'essence même du christianisme. C'est pourquoi il s'étonne qu'il n'en soit pas question lors d'un congrès de catholiques auquel il lui a été donné d'assister (congrès dont le souvenir lui inspirera le décor et certaines péripéties de Todo modo) :

Que le monde catholique [...] ait renoncé à utiliser « le crucifix comme objet contondant* » [...] me paraissait et me paraît un fait positif dont on doit se réjouir, mais à condition que le crucifix, cessant d'être une arme, soit une présence vivante et inquiétante, une passion, une « agonie ». L’agonie de Pascal : « Jésus sera en agonie jusqu’à la fin du monde et jusque-là on ne pourra plus dormir "; l'agonie d'Unamuno. Voilà : la fin du monde. Mais on n'en parlait pas. Voilà : la mort. Mais, en deux jours, je n'ai jamais entendu ce mot tomber, même incidemment, dans les discours qui se sont faits ${ }^{17}$.

Pour sa part, Sciascia a constamment à l'esprit la pensée de la mort ; non pas parce que cette mort, sa mort, l'angoisse personnellement. Non. Si la pensée de la mort lui paraît fondamentale, c'est qu'elle est la mesure et le terme, un temps déterminé justement, pendant lequel il convient d'être pleinement "vivant, au milieu de tant d'“âmes mortes" "8 ". C'est par là que, selon l'écrivain, la pensée de la mort est aussi une condition indispensable pour accéder au bonheur dont on a perdu jusqu'à la notion parce que, " en ayant perdu la notion de la mort, la pensée de la mort, on a perdu, en même temps, le sens de la vie ${ }^{19}$ ». En dehors de la pensée de la mort - à son propre usage, comme condition de la vie et non dans l'attente d'une autre vie -, Sciascia partage, ou semble partager, un autre sentiment avec les chrétiens : la pitié. Celle qu'il ressent pour Aldo Moro, par exemple ; celle qui lui fait dire qu'en écrivant L'affaire Moro il a écrit " un livre religieux. Le centre du livre est un sentiment de pitié pour cet homme seul, trahi, taxé de fou par ses propres amis ${ }^{20} »$.

I7. L. Sciascia, Nero su nero, Torino, Einaudi, I979, p. 56. * L'expression est de Gide.

18. Idem, La Sicile comme métaphore, p. 139 (p. 88, pour l'édition italienne).

19. Idem, La palma va al Nord, Roma, Ed. Quaderni radicali, 198I, p. 7I.

20. Ibidem, p. I44. 
Du moment où j'ai décidé d'écrire un livre sur l'affaire Moro [dit-il] et jusqu'à aujourd'hui, j'ai vécu et vis une expérience religieuse, comme un processus de réversibilité. Fils des Lumières, comme L'Unità m'étiquette, laïc et laïciste, incommensurablement éloigné du Moro catholique et du Moro démocrate-chrétien, j'ai ressenti religieusement le besoin de racheter Moro, prisonnier et victime des Brigades Rouges, de la vie où [on] l'a relégué ${ }^{21}$.

Relégué, Moro, dans la prison du peuple, parce que ses amis démocrates chrétiens ont découvert que dans leur chaîne de causalité à eux, dans l'historicisme absolu qui fait la grandeur de leur Église ${ }^{22}$, l'absence de l'onorevole Aldo Moro est plus profitable que sa présence. Relégué, fra Diego, chez les hérétiques pour avoir voulu donner une interprétation trop sociale de l'Évangile. Relégué, Monseigneur Ficcara, dans son archevêché fantôme de Leontopoli de Augustamnica, pour n'avoir voulu être que le pasteur de ses brebis chrétiennes. Et il semble bien que l'on puisse dire que c'est par cette relégation qui les rapproche qu'ils se rapprochent de Sciascia, qu'ils le rejoignent ou qu'il les rejoint. C'est parce qu'ils ont été exclus de l'Église du dieu de la force et parce qu'ils croient encore dans le Dieu caché - et seulement pour cela - que Sciascia peut voir en eux des coreligionnaires et des compatriotes, c'est-à-dire des hommes évangéliquement respectueux de la justice et de la vérité qui se retrouvent comme lui, et parfois malgré eux, du côté des infidèles, en toute ambiguïté sémantique, in partibus infidelium.

2I. Ibidem, p. 78 .

22. Cf. supra, note I6. 
